\title{
ON A CONJECTURE OF ATHANASIADIS RELATED TO FREENESS OF A FAMILY OF HYPERPLANE ARRANGEMENTS
}

\author{
TAKURo ABE
}

\begin{abstract}
We prove a characterization of freeness, conjectured by Athanasiadis, for the family of hyperplane arrangements, which lie between the Coxeter and the Catalan arrangement of the type $A_{\ell}$. One direction was already proved in [2]. Here we prove the other direction
\end{abstract}

\section{Conjecture of Athanasiadis}

The problem to characterize freeness has been an important and interesting one in the theory of hyperplane arrangements. For example, the freeness of graphic arrangements is characterized by Stanley in terms of chordal graphs (see [9] and [6] for details). Also, free arrangements between the Coxeter arrangements of the types $A_{\ell-1}$ and $B_{\ell}$ are characterized by Edelman and Reiner in [6], and those between the Coxeter and Shi arrangements of the type $A_{\ell}$ by Athanasiadis in [4]. A conjecture of Athanasiadis, which was introduced in [5], is a generalization of his freeness characterization in [4]. To state the conjecture, let us recall several definitions and results. In this paper, we use [8] as a general reference and the same notation as in [2].

Let $\mathbb{K}$ be an arbitrary field of characteristic zero and consider an affine arrangement in $V^{\ell+1}=\mathbb{K}^{\ell+1}$ defined by

$$
x_{i}-x_{j}=-a-\epsilon(i, j),-a,-(a-1), \ldots, a, a+\epsilon(j, i) \quad(1 \leq i<j \leq \ell+1),
$$

where $a \in \mathbb{Z}_{\geq 0}$ and $\epsilon(i, j)=0$ or 1 . Note that we distinguish $(i, j)$ and $(j, i)$ as explained later. Such arrangements are examples of deformations of the braid arrangement. To study the arrangement above, Athanasiadis introduced the directed graph $G$ consisting of the vertex set $V_{G}=\{1,2, \ldots, \ell+1\}$ and the set of directed edges $E_{G} \subset\{(i, j) \mid 1 \leq i, j \leq \ell+1, i \neq j\}$. Here the edge $(i, j)$ is the arrow from $i$ to $j$. If we define

$$
\epsilon(i, j):= \begin{cases}1, & \text { if }(i, j) \in E_{G}, \\ 0, & \text { if }(i, j) \notin E_{G},\end{cases}
$$

then there is a one-to-one correspondence between the deformations of the form (1.1) and the digraph above. In [3], Athanasiadis gave a product formula of the

Received by the editors October 19, 2011.

2000 Mathematics Subject Classification. Primary, 32S22.

Key words and phrases. braid arrangement, free (multi)arrangement, digraph, signed graph, signed eliminable graph, conjecture of Athanasiadis. 
characteristic polynomial of $\mathcal{A}_{G}$ when $G$ satisfies the following two conditions:

(A1) For every triple $i, j, k$ with $i, j<k$, it holds that, if $(i, j) \in E_{G}$, then either $(i, k) \in E_{G}$ or $(k, j) \in E_{G}$.

(A2) For every triple $i, j, k$ with $i, j<k$, it holds that, if $(i, k) \in E_{G}$ and $(k, j) \in E_{G}$, then $(i, j) \in E_{G}$.

Note that the conditions (A1) and (A2) depend on the ordering of the vertices $V_{G}$. Hence, we say a digraph $G$ satisfies (A1) and (A2) if the graph satisfies them after re-ordering the set of vertices $V_{G}$. Based on these results and definitions, Athanasiadis made the following conjecture.

Conjecture 1.1 ([5], Conjecture 6.6). Let $a=0$ in the deformation (1.1). Then the coning $c \mathcal{A}_{G}$ of $\mathcal{A}_{G}$ is free if and only if $G$ satisfies (A1) and (A2).

In [2], it was proved that conditions (A1) and (A2) are sufficient for Conjecture 1.1 in more general setting, i.e., the following holds.

Theorem 1.1 ([2], Theorem 5.3). In the deformation (1.1), $c \mathcal{A}_{G}$ is free if $G$ satisfies (A1) and (A2).

In this paper, we prove the converse of Theorem 1.1 as follows:

Theorem 1.2. In the deformation (1.1), if $c \mathcal{A}_{G}$ is free, then $G$ satisfies(A1) and (A2).

The conjecture of Athanasiadis is a corollary of Theorem 1.2.

Corollary 1.1. The Conjecture 1.1 of Athanasiadis is true.

In the next section, we prove Theorem 1.2. The idea of the proof is to lift up the signed eliminable ordering of the signed graph to that on the digraph, which makes $G$ satisfy (A1) and (A2). For the proof, we give a characterization of digraphs satisfying (A1) and (A2) independent of the numbering of vertices.

\section{Proof of Theorem 1.2}

To prove Theorem 1.2 we need Lemma 2.1 and Proposition 2.1.

Lemma 2.1. If a digraph $G$ contains one of the following induced subgraphs $H \subset G$ with $V_{H}=\{i, j, k\}$, then $c \mathcal{A}_{G}$ is not free:

(1) $E_{H}=\{(i, j),(j, k)\}$.

(2) $E_{H}=\{(i, j),(j, k),(k, i)\}$.

(3) $E_{H}=\{(i, j),(j, k),(k, i),(i, k)\}$.

Proof. Let $H_{\infty}$ denote the infinite hyperplane of $c \mathcal{A}_{G}$ added to $\mathcal{A}_{G}$. If $c \mathcal{A}_{G}$ is free, then every localization of it is also free. Also, if $H$ is an induced subgraph of $G$ corresponding to the set of vertices $V_{H}=\{i, j, k\} \subset V_{G}$, then $c \mathcal{A}_{H} \times \emptyset_{\ell-2}=\left(c \mathcal{A}_{G}\right)_{X}$, where $X=\left\{x_{i}=x_{j}=x_{k}\right\} \cap H_{\infty}$ and $\emptyset_{\ell-2}$ is an $(\ell-2)$-dimensional empty arrangement. Since the freeness of $c \mathcal{A}_{H} \times \emptyset_{\ell-2}$ is equivalent to that of $c \mathcal{A}_{H}$, we may assume that $\ell=2, i=1, j=2, k=3$ and check the non-freeness of the deformation of $A_{2^{-}}$ type arrangements. For that purpose, we check the characteristic polynomial of (1), (2) and (3). We can obtain them by [1], or direct computations. For $(1), \chi\left(\mathcal{A}_{G}, t\right)=$ $t\left(t^{2}-(6 a+5) t+\left(9 a^{2}+15 a+7\right)\right)$. For $(2), \chi\left(\mathcal{A}_{G}, t\right)=t\left(t^{2}-(6 a+6) t+\left(9 a^{2}+18 a+11\right)\right)$. For $(3), \chi\left(\mathcal{A}_{G}, t\right)=t\left(t^{2}-(6 a+7) t+\left(9 a^{2}+21 a+13\right)\right)$. Since they are irreducible over $\mathbb{Z}$, Terao's factorization theorem in [10] completes the proof. 
The second result is a characterization of digraphs satisfying (A1) and (A2) without using the numbering of vertices. To state it, let us introduce a correspondence map $S$ from a digraph $G=\left(V_{G}, E_{G}\right)$ to a signed graph $S(G):=\bar{G}=\left(V_{\bar{G}}, E_{+} \cup E_{-}\right)$with $E_{\mu} \subset\{\{i, j\} \mid 1 \leq i, j \leq \ell+1, i \neq j,\{i, j\}=\{j, i\}\}(\mu \in\{+,-\})$ and $E_{+} \cap E_{-}=\emptyset$. First, the sets of vertices are the same; $V_{G}=V_{\bar{G}}$. The relation between edges are as follows:

- If $(i, j) \in E_{G}$ and $(j, i) \in E_{G}$, then $\{i, j\} \in E_{+}$.

- If exactly one of $(i, j)$ and $(j, i)$ belongs to $E_{G}$, then $\{i, j\} \notin E_{+} \cup E_{-}$.

- If $(i, j) \notin E_{G}$ and $(j, i) \notin E_{G}$, then $\{i, j\} \in E_{-}$.

Also, recall the definition of a signed eliminable graph introduced in [2]. We say a signed graph $\left(V_{\bar{G}}, E_{+} \cup E_{-}\right)$is signed eliminable if there exists a numbering $\{1,2, \ldots, \ell+1\}$ of $V_{\bar{G}}$ such that for any $i, j, k \in V_{\bar{G}}$ with $i, j<k$, the following two conditions are satisfied:

(SE1) If $\{k, i\} \in E_{\mu}$ and $\{i, j\} \in E_{\nu}$ for $\{\mu, \nu\}=\{+,-\}$, then $\{k, j\} \in E_{\nu}$.

(SE2) If $\{k, i\} \in E_{\mu}$ and $\{k, j\} \in E_{\mu}$ for $\mu \in\{+,-\}$, then $\{i, j\} \in E_{\mu}$.

For details, see [2] and [7]. Note that a characterization of a signed eliminable graph without using a numbering of vertices is given in [7].

Proposition 2.1. Let $G$ be a digraph and $S(G)=\bar{G}$ a signed graph defined above. Then $G$ satisfies (A1) and (A2) if and only if $\bar{G}$ is signed eliminable and $G$ does not contain any induced subgraph of three-vertices (1),(2) and (3) in Lemma 2.1.

Proof. First assume that $G$ satisfies (A1) and (A2). Then it was proved in [2] that the corresponding signed graph $S(G)=\bar{G}$ is signed eliminable. Also, by definition, any induced subgraph of three vertices of $G$ satisfies (A1) and (A2). Since any numbering on vertices of digraphs (1), (2) and (3) in Lemma 2.1 cannot make them satisfy (A1) and (A2), $G$ cannot contain any of these subgraphs.

Next assume that $\bar{G}$ is signed eliminable and $G$ does not contain any of (1), (2) and (3) in Lemma 2.1. We may assume that $1,2, \ldots, \ell+1$ is a signed elimination ordering. We claim that the same ordering on $V_{\bar{G}}=V_{G}$ makes $G$ into a digraph satisfy (A1) and (A2).

To check this claim, it suffices to check that, for an induced subgraph $\bar{H}=$ $\{i, j, k\} \subset V_{\bar{G}}$ of $\bar{G}$ with $i, j<k$, the induced subgraph $H:=\{i, j, k\} \subset V_{G}$ of $G$ satisfies two conditions (A1) and (A2). Let us denote $\bar{H}=\left(\{i, j, k\},\left.\left.E_{+}\right|_{\bar{H}} \cup E_{-}\right|_{\bar{H}}\right)$. This lifting correspondence from $\bar{H}$ to $H$ is as follows:

- If $\left.\{i, j\} \in E_{+}\right|_{\bar{H}}$, then $(i, j) \in E_{G}$ and $(j, i) \in E_{G}$.

- If $\left.\left.\{i, j\} \notin E_{+}\right|_{\bar{H}} \cup E_{-}\right|_{\bar{H}}$, then either $(i, j) \in E_{G}$ or $(j, i) \in E_{G}$.

- If $\left.\{i, j\} \in E_{-}\right|_{\bar{H}}$, then $(i, j) \notin E_{G}$ and $(j, i) \notin E_{G}$.

So there are only finite possibilities of the induced subgraph $H$ of $G$ as a lifting of $\bar{H}$. Let us check the lifting $H$ of $\bar{H}$ in every case. Put $\left.E\right|_{\bar{H}}:=\left.\left.E_{+}\right|_{\bar{H}} \cup E_{-}\right|_{\bar{H}}$.

Case 1. $\left.E\right|_{\bar{H}}=\emptyset$. In this case, using symmetry, we have four possibilities of $H$ :

(1) $\{(i, j),(i, k),(k, j)\}=E_{H}$.

(2) $\{(i, j),(i, k),(j, k)\}=E_{H}$.

(3) $\{(i, j),(k, i),(k, j)\}=E_{H}$.

(4) $\{(i, j),(k, i),(j, k)\}=E_{H}$. 
In these four cases, only (4) does not satisfy (A1) and (A2), which cannot occur by assumption.

Case 2. $\left|E_{+}\right|_{\bar{H}} \mid=1$ and $\left.E_{-}\right|_{\bar{H}}=\emptyset$. In this case, using symmetry, we have seven possibilities of $H$ :

(1) $\{(i, j),(j, i),(i, k),(k, j)\}=E_{H}$.

(2) $\{(i, j),(j, i),(i, k),(j, k)\}=E_{H}$.

(3) $\{(i, j),(j, i),(k, i),(k, j)\}=E_{H}$.

(4) $\{(i, j),(k, i),(i, k),(k, j)\}=E_{H}$.

(5) $\{(j, i),(k, i),(i, k),(k, j)\}=E_{H}$.

(6) $\{(i, j),(k, i),(i, k),(j, k)\}=E_{H}$.

(7) $\{(j, i),(k, i),(i, k),(j, k)\}=E_{H}$.

In these seven cases, (1), (5) and (6) do not satisfy (A1) and (A2), which cannot occur by assumption.

Case 3. $\left.E_{+}\right|_{\bar{H}}=\emptyset$ and $\left|E_{-}\right|_{\bar{H}} \mid=1$. In this case, using symmetry, we have seven possibilities of $H$ :

(1) $\{(i, k),(k, j)\}=E_{H}$.

(2) $\{(i, k),(j, k)\}=E_{H}$.

(3) $\{(k, i),(k, j)\}=E_{H}$.

(4) $\{(i, j),(j, k)\}=E_{H}$.

(5) $\{(i, j),(k, j)\}=E_{H}$.

(6) $\{(j, i),(j, k)\}=E_{H}$.

(7) $\{(j, i),(k, j)\}=E_{H}$.

In these seven cases, (1), (4) and (7) do not satisfy (A1) and (A2), which cannot occur by assumption.

Case 4. $\left|E_{+}\right|_{\bar{H}} \mid=2$ and $\left.E_{-}\right|_{\bar{H}}=\emptyset$. In this case, recall that $\bar{G}$ is, and so $\bar{H}$ is signed eliminable. Hence we can restrict the possibility of $H$ and $\bar{H}$. For example,

$$
\{(i, j),(i, k),(k, i),(k, j),(j, k)\}=E_{H}
$$

cannot occur because $\left.E_{+}\right|_{\bar{H}}=\{\{i, k\},\{k, j\}\}$ and $\left.E_{-}\right|_{\bar{H}}=\emptyset$ are not permitted by the signed eliminability. Taking into account the fact that $\bar{G}$ is signed eliminable, using symmetry, we have two possibilities of $H$ :

(1) $\{(i, j),(j, i),(i, k),(k, i),(j, k)\}=E_{H}$.

(2) $\{(i, j),(j, i),(i, k),(k, i),(k, j)\}=E_{H}$.

Both cases satisfy (A1) and (A2).

Case 5. $\left.E_{+}\right|_{\bar{H}}=\emptyset$ and $\left|E_{-}\right|_{\bar{H}} \mid=2$. In this case, taking into account the fact that $\bar{G}$ is signed eliminable, using symmetry, we have two possibilities of $H$ :

(1) $\{(i, k)\}=E_{H}$.

(2) $\{(k, i)\}=E_{H}$.

Both cases satisfy (A1) and (A2).

Case 6. $\left|E_{+}\right|_{\bar{H}} \mid=1$ and $\left|E_{-}\right|_{\bar{H}} \mid=1$. In this case, taking into account the fact that $\bar{G}$ is signed eliminable, using symmetry, we have two possibilities of $H$ :

(1) $\{(i, j),(i, k),(k, i)\}=E_{H}$.

(2) $\{(j, i),(i, k),(k, i)\}=E_{H}$. 
Both cases satisfy (A1) and (A2).

Case 7. $\left|E_{+}\right|_{\bar{H}} \mid=3$ and $\left.E_{-}\right|_{\bar{H}}=\emptyset$. In this case, we have only one possibility of $H$ :

(1) $\{(i, j),(j, i),(k, i),(i, k),(j, k),(k, j)\}=E_{H}$.

This digraph satisfies (A1) and (A2).

Case 8. $\left.E_{+}\right|_{\bar{H}}=\emptyset$ and $\left|E_{-}\right|_{\bar{H}} \mid=3$. In this case, $H$ has no edges. Hence, it satisfies (A1) and (A2).

Case 9. $\left|E_{+}\right|_{\bar{H}} \mid=2$ and $\left|E_{-}\right|_{\bar{H}} \mid=1$. In this case, taking into account the fact that $\bar{G}$ is signed eliminable, using symmetry, we have only one possibility of $H$ :

(1) $\{(i, j),(j, i),(k, i),(i, k)\}=E_{H}$.

This satisfies (A1) and (A2).

Case 10. $\left|E_{+}\right|_{\bar{H}} \mid=1$ and $\left|E_{-}\right|_{\bar{H}} \mid=2$. In this case, taking into account the fact that $\bar{G}$ is signed eliminable, using symmetry, we have only one possibility of $H$ :

(1) $\{(i, k),(k, i)\}=E_{H}$.

This satisfies (A1) and (A2).

The classification above shows that the signed elimination ordering of $\bar{G}$ makes $G$ into a digraph satisfying (A1) and (A2), which completes the proof.

Proof of Theorem 1.2. Let $G=\left(V_{G}, E_{G}\right)$ be a digraph and $\mathcal{A}_{G}$ the corresponding deformation of a braid arrangement of the form (1.1). Assume that $c \mathcal{A}_{G}$ is free. Take the Ziegler restriction of $c \mathcal{A}_{G}$ onto the infinite hyperplane $H_{\infty} \in c \mathcal{A}_{G}$. Then we obtain a multiarrangement $\left(\mathcal{A}_{\ell}, m_{\bar{G}}\right)$, where $\mathcal{A}_{\ell}$ is a braid arrangement in $\mathbb{K}^{\ell+1}, S(G)=\bar{G}=$ $\left(V_{G}, E_{+} \cup E_{-}\right)$is a signed graph defined above and $m_{\bar{G}}$ is the corresponding multiplicity to $\bar{G}$. See [2] for details. By [11], the Ziegler restriction of a free central arrangement is a free multiarrangement. Hence $\left(\mathcal{A}_{\ell}, m_{\bar{G}}\right)$ is free. Then Theorem 0.3 in [2] shows that $\bar{G}$ is signed eliminable. For any codimension three flat $X \in L\left(c \mathcal{A}_{G}\right)$ with $X \subset H_{\infty}$, the localization $\left(c \mathcal{A}_{G}\right)_{X}$ is free since $c \mathcal{A}_{G}$ is free. Let $X=\left\{x_{i}=x_{j}=x_{k}\right\} \cap H_{\infty}$. Then $\left(c \mathcal{A}_{G}\right)_{X}$ is a deformation of $A_{2}$-type arrangements. Also, by definition of the deformation (1.1), there is an induced subgraph $H$ of $G$ with $V_{H}=\{i, j, k\}$ such that $\left(c \mathcal{A}_{G}\right)_{X}=c \mathcal{A}_{H} \times \emptyset_{\ell-2}$. Then freeness of $\left(c \mathcal{A}_{G}\right)_{X}=c \mathcal{A}_{H} \times \emptyset_{\ell-2}$ and Lemma 2.1 show that the subgraphs (1), (2) and (3) in Lemma 2.1 are not contained in $G$. Then Proposition 2.1 implies that $G$ satisfies (A1) and (A2).

\section{Acknowledgments}

The author is grateful to Christos Athanasiadis for helpful comments to this article. The author is partially supported by JSPS Grants-in-Aid for Young Scientists (B) no. 21740014 .

\section{References}

[1] T. Abe, The stability of the family of $A_{2}$-type arrangements, J. Math. Kyoto Univ. 46(3) (2006), $617-639$.

[2] T. Abe, K. Nuida and Y. Numata, Signed eliminable graphs and free multiplicities on the braid arrangement, J. Lond. Math. Soc. 80(1) (2009), 121-134.

[3] C. A. Athanasiadis, Characteristic polynomials of subspace arrangements and finite fields, Adv. Math. 122 (1996), 193-233. 
[4] C. A. Athanasiadis, On free deformations of the braid arrangement, Eur. J. Combin. 19 (1998), $7-18$.

[5] C. A. Athanasiadis, Deformations of Coxeter hyperplane arrangements and their characteristic polynomials, in Arrangements - Tokyo 1998. 1-26, Adv. Stud. Pure Math. 27, Kinokuniya, Tokyo, 2000.

[6] P. H. Edelman and V. Reiner, Free hyperplane arrangements between $A_{n-1}$ and $B_{n}$, Math. Z. 215 (1994), 347-365.

[7] K. Nuida, A characterization of signed graphs with generalized perfect elimination orderings, Discrete Math. 310(4) (2010), 819-831.

[8] P. Orlik and H. Terao, Arrangements of hyperplanes, Grundlehren der Mathematischen Wissenschaften, 300. Springer-Verlag, Berlin, 1992.

[9] R. P. Stanley, Supersolvable lattices, Algebr. Univ. 2 (1972), 197-217.

[10] H. Terao, Generalized exponents of a free arrangement of hyperplanes and Shephard-ToddBrieskorn formula, Invent. Math. 63 (1981), 159-179.

[11] G. M. Ziegler, Multiarrangements of hyperplanes and their freeness, in Singularities (Iowa City, IA, 1986), 345-359, Contemp. Math., 90, Amer. Math. Soc., Providence, RI, 1989.

Department of Mechanical Engineering and Science, Kyoto University, Yoshida Hon-Machi SAKYO-KU, KyOTO 606-8501, JAPAN

E-mail address: abe.takuro.4c@kyoto-u.ac.jp 